\title{
Simultaneous Assessment of Homonymous and Heteronymous Monosynaptic Reflex Excitability in the Adult Rat
}

\author{
(1) Calvin C. Smith, ${ }^{1}$ Doger W. P. Kissane, ${ }^{2}$ and ${ }^{-}$Samit Chakrabarty ${ }^{2}$
}

https://doi.org/10.1523/ENEURO.0227-18.2018

\begin{abstract}
${ }^{1}$ Department of Neuromuscular Diseases, UCL Queen Square Institute of Neurology, University College London, London, United Kingdom, WC1N 3BG, and ${ }^{2}$ School of Biomedical Sciences, Faculty of Biological Sciences, University of Leeds, Leeds, United Kingdom, LS2 9JT
\end{abstract}

\begin{abstract}
In order to successfully perform motor tasks such as locomotion, the central nervous system must coordinate contractions of antagonistic and synergistic muscles across multiple joints. This coordination is largely dependent upon the function of proprioceptive afferents (PAs), which make monosynaptic connections with homonymous motoneurons. Homonymous pathways have been well studied in both health and disease but their collateral fibers projecting to heteronymous, synergistic muscles receive relatively less attention. This is surprising given that PA collaterals have significant effects on the excitability of heteronymous motoneurons, and that their synaptic terminal density is activity dependent. It is likely that the relative lack of literature is due to the lack of a preparation which allows synergistic heteronymous pathways to be assessed in vivo. Here, we describe a method to simultaneously evoke homonymous and heteronymous (synergistic) monosynaptic reflexes (MSRs) and study their modulation by descending pathways in adult rats. Through stimulation of the medial plantar nerve, we were able to produce an $\mathrm{H}$ reflex in the intrinsic foot (IF) muscles of the hind paw with a latency of $10.52 \pm 3.8 \mathrm{~ms}$. Increasing the stimulus intensity evoked a robust signal with a monosynaptic latency (11.32 $\pm 0.35 \mathrm{~ms})$, recorded in the ipsilateral gastrocnemius (Gs). Our subsequent analyses suggest that Gs motoneurons were activated via heteronymous afferent collaterals from the medial plantar nerve. These reflexes could be evoked bilaterally and were modulated by conditioning stimuli to the cortex $(\mathrm{Cx})$ and reticular formation. Interestingly, cortical stimulation was equally efficient at modulating both ipsilateral and contralateral reflexes, indicating that cortical modulation of lumbar sensory afferents lacks the laterality demonstrated by studies of cortical muscle activation. This technique represents a novel, relatively simple way to assess heteronymous afferent pathways in normal motor control as well as in models of motor disorders where adaptive and maladaptive plasticity of PAs and descending systems affects functional outcomes.
\end{abstract}

Key words: Monosynaptic reflex; Homonymous; Heteronymous; modulation; corticospinal; reticulospinal

\section{Significance Statement}

This study describes a method for simultaneously recording bilateral homonymous and heteronymous $\mathrm{PA}$ reflexes in rodents. Bilateral stimulation of the medial plantar nerve produced homonymous $\mathrm{H}$ reflexes in IF muscles of both hind paws as well as heteronymous MSR in the Gs muscles. We used this technique to assess cortical and reticular modulation of homonymous and heteronymous reflexes. Surprisingly, cortical conditioning of reflexes revealed a lack of laterality in modulation of homonymous and heteronymous reflexes. This technique will be extremely useful for further studies of heteronymous afferent pathways in normal and dysfunctional motor states, such as spinal cord injury, stroke, and cerebral palsy. 


\section{Introduction}

To successfully perform motor tasks such as locomotion, the CNS must coordinate contractions of antagonistic and synergistic muscles across multiple joints. Previous work demonstrates that sensory information conveyed by peripheral receptors and their afferent fibers are essential to ensuring precise movement control (Pearson, 1995). The importance of sensory information to movement control increases following lesions to the descending control systems, in conditions such as spinal cord injury and cerebral palsy (Takeoka et al., 2014). While sensory afferents have been shown to be crucial to recovery of locomotor function, they are also implicated in development of dysfunctional states such as spasticity (Tan et al., 2012; Smith et al., 2017). So far, our understanding of these outcomes is based primarily on assessment of PA connections (and their modulation) between muscle spindles and motoneurons of the same (homonymous) muscle. However, PAs are known to make many projections to other, mainly synergistic motor pools (Eccles et al., 1957; Nelson and Mendell, 1978). These heteronymous connections are thought to be extremely important for establishing and coordinating muscle synergies to ensure biomechanical efficiency and stability of limb trajectories (Burkholder and Nicols, 2000). Despite this knowledge, synergistic PA collaterals are rarely studied in either normal or dysfunctional motor control. Considering the aberrant recruitment of muscle synergies in spastic and dystonic conditions, which is often sensory induced, the plasticity of these heteronymous connections and their modulation may be important mechanisms underlying motor dysfunction (Nielsen et al., 2007). This hypothesis is supported by the fact that PA terminal density on heteronymous, synergistic motoneurons is activity dependent (Mendelsohn et al., 2015).

The development of new techniques is crucial for furthering our understanding of neurophysiology in health and disease. The relative lack of studies assessing PA collateral projections to heteronymous motoneurons likely reflects the absence of a preparation permitting electrophysiological access to the circuit in vivo. Here, we describe a method by which stimulating the medial plantar nerves simultaneously evokes homonymous MSRs in the IF muscles and heteronymous MSRs from the Gs muscle. We characterize these reflexes in terms of their recruit-

Received June 7, 2018; accepted September 16, 2018; First published October 01, 2018

The authors declare no competing financial interests.

Author contributions: C.C.S. and S.C. designed research; C.C.S., R.W.K., and S.C. performed research; S.C. contributed unpublished reagents/analytic tools; C.C.S., R.W.K., and S.C. analyzed data; C.C.S., R.W.K., and S.C. wrote the paper.

This work was supported by the International Federation for Research in Paraplegia Grant P-153.

Correspondence should be addressed to Samit Chakrabarty at the above address, E-mail: s.chakrabarty@leeds.ac.uk.

https://doi.org/10.1523/ENEURO.0227-18.2018

Copyright (C) 2018 Smith et al.

This is an open-access article distributed under the terms of the Creative Commons Attribution 4.0 International license, which permits unrestricted use, distribution and reproduction in any medium provided that the original work is properly attributed. ment and paired pulse interaction profiles. Additionally, we assessed descending modulation of these reflexes by delivering conditioning stimuli to either the motor cortex or medial reticular formation (MRF).

The work describes a novel, relatively simple technique for assessing plasticity of homonymous and heteronymous afferents and their modulation by descending systems. These methods will be important for future study of sensory control of movement, especially in movement disorders such as spinal cord injury.

\section{Materials and Methods}

Experiments and procedures were performed in a manner that conformed to the United Kingdom Animals (Scientific Procedures) Act 1986. Approval was granted by the local Animal Welfare and Ethics committee (University of Leeds).

\section{Animals}

Male Wistar rats $(339 \pm 21 \mathrm{~g})$ were used in all experiments. Number of animals for each experiment is indicated in Results.

\section{Preparation}

Initial anesthesia was induced by intraperitoneal administration of a ketamine $(100 \mathrm{mg} / \mathrm{kg})$ and xylazine $(5 \mathrm{mg} / \mathrm{kg})$ $\mathrm{mix}$, and the anesthetic plain maintained through intramuscular injections of ketamine $(50 \mathrm{mg} / \mathrm{kg}$ ) every $20-30$ min. The animal was continuously checked for nociceptive reflexes and additional anesthetic administered when required. On loss of paw withdrawal, the hind limbs and top of the head were shaved. The animal was placed prone in a custom stereotaxic frame and the head was fixed in place with ear bars and mouthpiece. A heating pad maintained the animal temperature at $37^{\circ} \mathrm{C}$.

\section{Simultaneous stimulation and recording of homonymous Intrinsic foot $\mathrm{H}$ reflex and heteronymous gastrocnemius MSR}

Bilateral, subcutaneous bipolar needle electrodes (SpesMedica) were inserted adjacent to medial malleolus to stimulate medial plantar divisions of the tibial nerve. A pair of insulated fine copper wires (40 AWG; $79 \mu \mathrm{m}$ in diameter) with 1.5- to 2-mm bared tips were inserted bilaterally into plantar IF muscles using a hypodermic needle $(27 \mathrm{G})$. These wires recorded the homonymous $\mathrm{H}$ reflex. A pair of multi-stranded stainless-steel wires (Cooner), bared by 1.5-2 mm near the tip, were inserted bilaterally into the gastrocnemius/soleus muscles. These wires recorded the heteronymous MSR. The hind limbs rested naturally on the heating pad, with the ankle angle at $\sim 100^{\circ}$. To evoke homonymous $\mathrm{H}$ reflexes, square pulse current $(0.3 \mathrm{~ms})$ was delivered from a stimulator (ISOSTIM 01M Stimulus Isolator Module, npi electronic) driven by a Master-8 (A.M.P.I.) every 5 s. Thresholds were identified as the stimulation intensity at which the smallest visible response occurred at a frequency of $50 \%$. Both IF $\mathrm{H}$ reflexes and Gs MSR were recorded simultaneously during graded stimulation of the medial plantar nerve until maximal IF M wave and Gs MSR were recorded (to produce a recruitment curve). Frequency-dependent depres- 


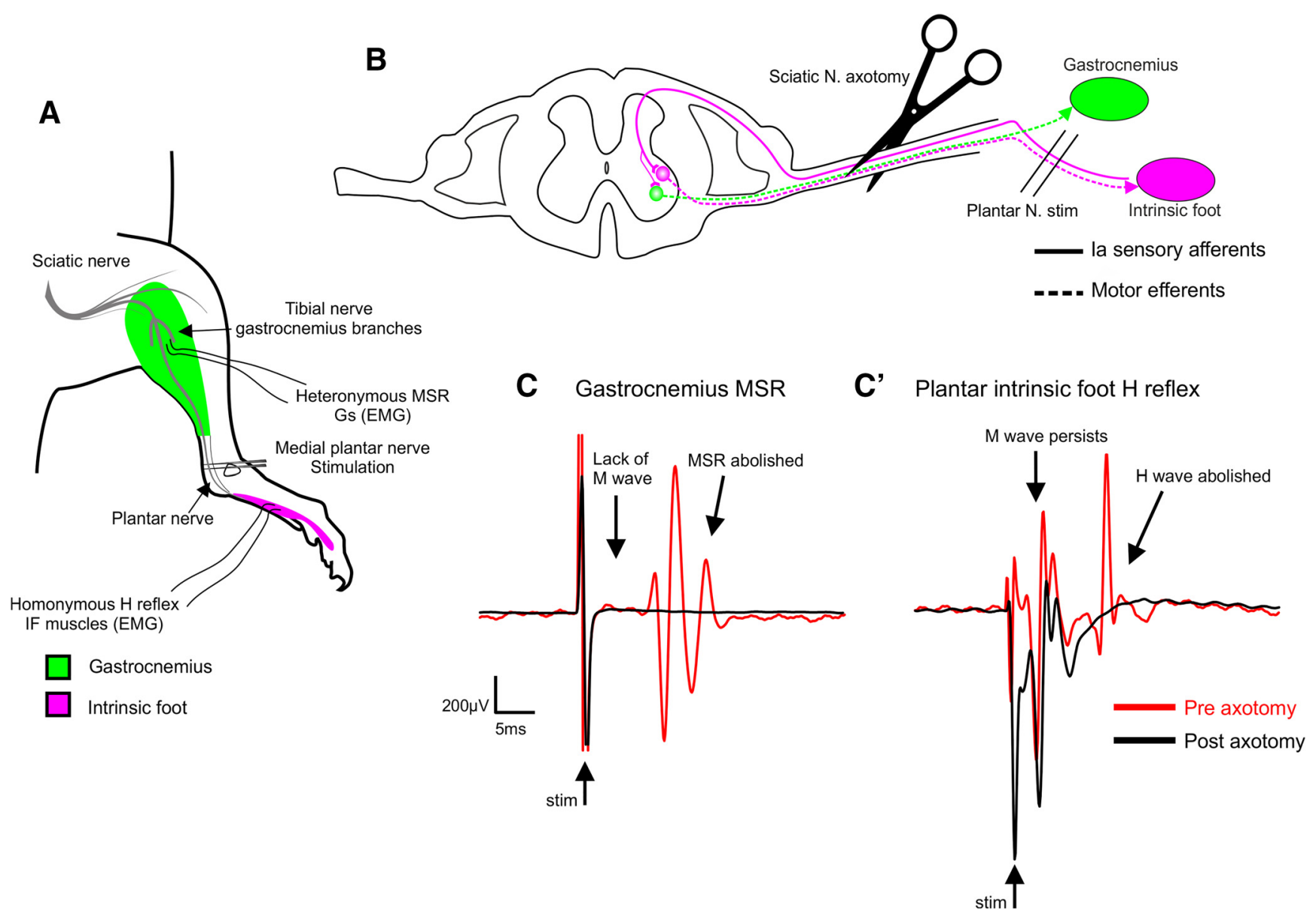

Figure 1. Experimental procedure for evoking homonymous $\mathrm{H}$ reflex and heteronymous MSR in IF and Gs muscles respectively. $\boldsymbol{A}$, Bipolar stimulation needles inserted at the medial maleolus, adjacent to the medial plantar nerve. Bipolar recording wire electrodes are inserted into the Gs and IF muscles. B, Graphical representation of homonymous/heteronymous pathways and sciatic nerve transection for confirmation of synaptic signal. Solid lines represent monosynaptic PA pathway with collateral from IF PA to Gs motoneuron. Dashed lines represent motor efferents. C, C', Traces showing Gs heteronymous MSR and homonymous IF H reflex before (red line) and after (black line) sciatic nerve axotomy. Notice that both late events are lost following axotomy suggesting they invole a synaptic component, whereas the early "M wave" remains. Double lines at the end of a stimulus artifact represent truncation for visual purposes.

sion/facilitation of the IF H reflex/Gs MSR was assessed by delivering paired pulses to the medial plantar nerve at intervals ranging from 1000 to 20 ms. For all conditioning experiments, IF H reflexes and Gs MSR were evoked on the ascending portion of their recruitment curves, as recorded at the beginning of each experiment $(1.0-1.5 \times$ threshold). This ensured that both facilitation and inhibition are possible (Crone et al., 1990; Knikou, 2008). Control (single stimulus) trials were performed immediately before test (double stimuli) for each time interval. This enabled us to express the test amplitudes as a percentage of the control and account for any changes in baseline MSR amplitudes occurring over time. At the end of some experiments, the sciatic nerve was transected proximal to the stimulation site. The late wave was confirmed unequivocally as synaptic ( $\mathrm{H}$ wave or MSR) if it was abolished after axotomy (Fig. $1 C, C^{\prime}$ ). The early $M$ wave always remained post transection as this signal results from direct motor axon conduction.

\section{Cortical and reticular conditioning stimuli}

Once the head was fixed in the stereotaxic frame using ear bars and mouthpiece, a midline incision was made on the skin covering the top of the skull. The skin was retracted and fascia removed from the skull. A $5 \times 5 \mathrm{~mm}$ area of the skull over the motor cortex was thinned using a drill and the skull flap removed using forceps. The dura was then incised and retracted from the window. Petroleum jelly was used to build a wall around the skull window so that warmed paraffin oil could bath the exposed cortex, preventing desiccation. Using a micromanipulator (Narishige), a stainless steel microelectrode (Microprobes) was lowered $1.5 \mathrm{~mm}$ into the ankle flexor region of the motor cortex (Neafsey et al., 1986). Monopolar stimulation was performed between the microelectrode and a ground clip attached to the skin of the scalp. Square wave pulses with a duration of $0.3 \mathrm{~ms}$ were delivered at a frequency of $300 \mathrm{~Hz}$. Five pulses were used to evoke a cortical motor-evoked potential (MEP) in the gas- 
trocnemius muscle, at which point the stimulation was reduced to sub-motor threshold. Only three pulses were used for conditioning stimuli. If triceps surae were not the primary muscle group activated, the position of the cortical electrode was adjusted. Sub-threshold stimuli were defined as maximum intensities at which no MEP was observed after 10 consecutive trials separated by $5 \mathrm{~s}$. Cortical conditioning stimuli were delivered $20 \mathrm{~ms}$ before medial plantar nerve stimulation as this was the latency to cortically evoked MEPs. Control IF reflexes were recorded, followed by cortical conditioning of IF MSRs; this was completed for both contralateral and ipsilateral sides by simply switching the stimulation to the contralateral plantar nerve. For conditioning experiments, IF H reflexes and Gs MSRs were evoked on the ascending portion of their recruitment curve so that both facilitation and inhibition were possible. Although IF $\mathrm{H}$ reflexes were also present during Gs MSRs, these responses were not quantified, as they were likely to be on the descending portion of their recruitment curve.

Coordinates from the Paxinos rat atlas were used to stimulate the MRF; however, stimulation sites were used for sub-threshold conditioning if they elicited a clear hindlimb response. Motor responses to MRF stimulation varied. We saw activation of all four limbs, both hindlimbs only, both forelimbs only, or a combination of hindlimbs and forelimbs. Due to variations in anatomic landmarks between animals, it was not possible to reproduce outputs between animals when specific coordinates were used. Therefore, a range of coordinates were used. In the rostro-caudal axis, stimulation sites were between 1 and $3.5 \mathrm{~mm}$ caudal to the interaural line (IA). The medio-lateral range was $1-2.5 \mathrm{~mm}$ lateral from midline. The depth of the tip of the electrode was $7-9 \mathrm{~mm}$ below the dura. We predict that most of the conditioning stimulation sites were within the gigantocellular nucleus of the reticular formation, however at depths of 7-8 mm, the MLF was also likely to be activated.

\section{Analysis}

All signals were recorded with a preamplifier (Digitimer) and sampled with Signal 5 (CED) software. Quantification of reflex amplitudes were made from averages of 15-30 traces which were generated using Signal 5. Depression of the MSR in response to homonymous paired pulse stimuli was calculated by expressing the test reflex as a percentage of control reflex. Averaged reflexes evoked after a conditioning stimulus to either cortex or reticular formation were compared to control trials which the reflex was evoked with no conditioning. A reflex was facilitated if the average amplitude was $>10 \%$ compared to control, or if the threshold of the response was reduced (Lundberg et al., 1962). The threshold was lowered if the addition of conditioning stimuli resulted in consistent reflex responses that were not observed in the absence of conditioning (sub-threshold afferent stimulation). A reflex response was inhibited if its amplitude was reduced by $10 \%$ or greater compared to the control average. All values are expressed as SEM.

\section{Results}

\section{Medial plantar nerve stimulation evokes homonymous $\mathrm{H}$ reflex in Intrinsic foot muscles and heteronymous MSR in gastrocnemius via afferent collaterals}

To simultaneously evoke homonymous IF muscle and heteronymous Gs MSRs, we stimulated the medial plantar nerve and recorded from both IF and Gs muscles (Fig. $1 A, B)$. There were two signals detected in the IF muscles. The first (M wave) occurred at $2.84 \pm 0.16 \mathrm{~ms}$ and the second $(H$ wave) occurred at $10.52 \pm 3.8 \mathrm{~ms}(n=12)$, representing the two major components of the $\mathrm{H}$ reflex (Fig. 1C'; Meinck, 1976). In the Gs muscles, there was a solitary late signal at $11.32 \pm 0.35 \mathrm{~ms}(n=10$; Fig. $1 C)$. There could have been several explanations for the stimulus evoked signal detected during simultaneous EMG recording from Gs. First, because the medial plantar nerve is a branch of the tibial nerve, which innervates Gs muscle proximally, we wanted to rule out electrotonic spread of current to Gs motor and afferent fibers in the tibial nerve. Gs motor nerve activation ( $M$ wave) was ruled out because the latency was too long (IF M wave $=2.84 \pm 0.16$, $n=12$ versus Gs response $=11.32 \pm 0.35 \mathrm{~ms}, n=10$ ), and the response was abolished on transection of the sciatic nerve, suggesting a synaptic pathway (Fig. 1B). A synaptic response could result from either activation of Gs afferent fibers in the tibial nerve or heteronymous intraspinal collaterals from the plantar nerve projecting to Gs motoneurones (Eccles et al., 1957). The latency of the Gs response was $1.18 \pm 0.12 \mathrm{~ms}$ later than the IF $\mathrm{H}$ reflex and the Gs threshold $(20.12 \pm 3.88 \mu \mathrm{A})$ was $2.38 \pm 0.36$ times greater than IF $\mathrm{H}$ reflex threshold $(8.63 \pm 1.20 \mu \mathrm{A})$. If the Gs response resulted from activation of homonymous Gs afferents, the latency would be earlier than the more distal IF muscle. Moreover, increasing stimulation intensity would eventually result in activation of motor fibers in the tibial nerve, yet we never observed an early $M$ response in the Gs muscle, even at stimulation intensities of $100 \mu \mathrm{A}$ (Fig. 1C).

\section{Comparing intrinsic foot $\mathrm{H}$ reflex to heteronymous gastrocnemius MSR \\ Recruitment curves}

We performed graded stimulation of the medial plantar nerve to establish recruitment profiles for both the $\mathrm{H}$ reflex and heteronymous Gs MSR. For the $\mathrm{H}$ reflex recruitment curve, the $H$ wave was expressed as a ratio of the $M$ wave and showed a classical profile (Knikou, 2008). In contrast to the $\mathrm{H}$ wave, the heteronymous Gs MSR increased with stimulation intensity up to its maximum value and was not reduced by further increases (Fig. $2 \mathrm{C}-H$ ). This is due to a lack of $M$ wave for the heteronymous reflex, meaning the synaptic response is not subject to antidromic collisions.

\section{Paired pulse interactions}

In response to paired stimuli, the $\mathrm{H}$ reflex classically shows frequency-dependent depression, which becomes complete at high frequencies (Ho and Waite, 2002; Tan et al., 2012; Kathe et al., 2016). The depression seen at lower frequency paired stimuli is thought to be due to both 

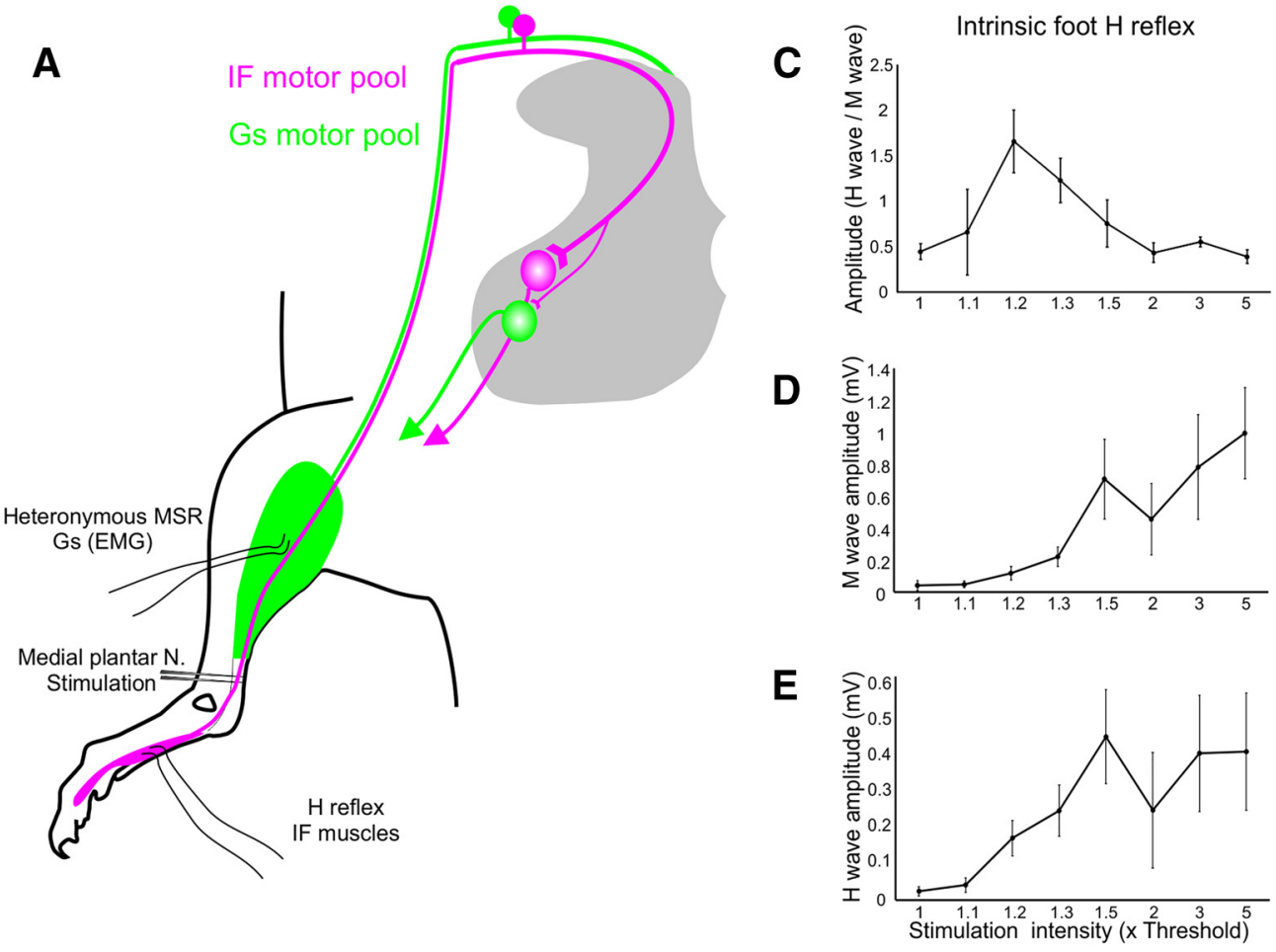

B
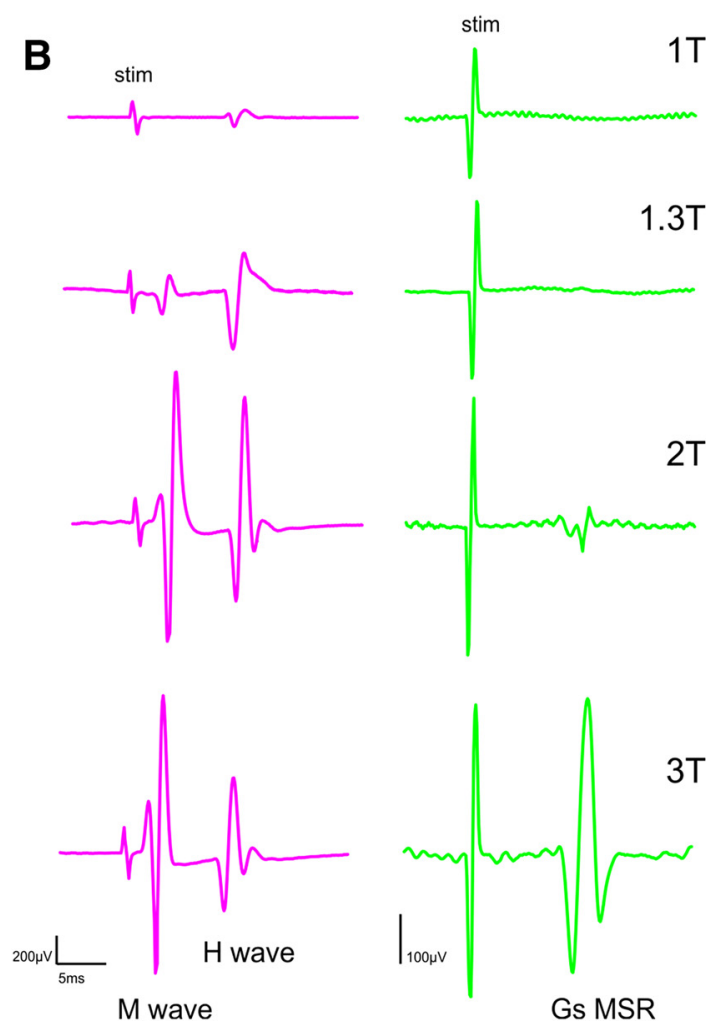

$\mathbf{F}$

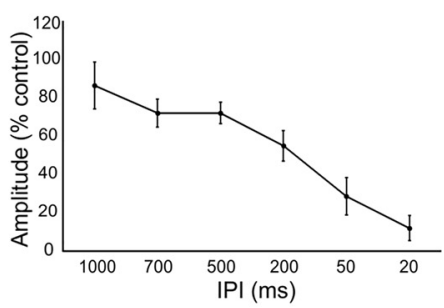

G

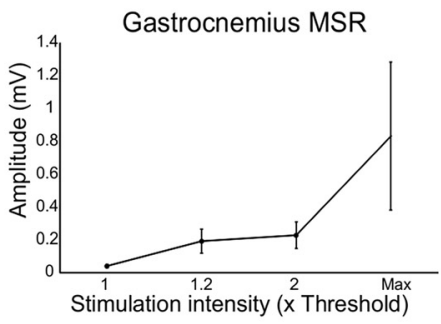

H

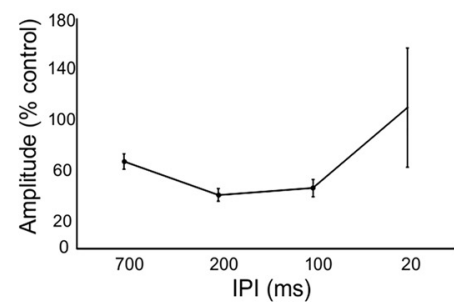

Figure 2. Characterization of homonymous IF H reflex alongside heteronymous Gs MSR. $\boldsymbol{A}$, Schematic of experimental set up. $\boldsymbol{B}$, Example of simultaneous EMG recording from IF (left) and Gs (right) during graded stimulation of the medial plantar nerve. $\boldsymbol{C}-\boldsymbol{F}$, Recruitment characteristics of the $\mathrm{H}$ reflex and paired pulse interactions. $\mathbf{G}-\boldsymbol{H}$, Heteronymous Gs recruitment curve and paired pulse interactions. 
inhibitory mechanisms as well availability of neurotransmitter following the previous la terminal activation, and is therefore an important assay for assessing synaptic efficacy and modulation of afferent fibers (Hultborn et al., 1996; Mende et al., 2016; Smith et al., 2017).

To further characterize the heteronymous Gs reflex, we compared the responses to conditioning pulses to the same nerve at several different time intervals. In agreement with previous literature, the IF $\mathrm{H}$ reflex experienced homosynaptic depression with increasing magnitude as stimulation interval times were reduced. For example, at 200 -ms depression was $54.88 \pm 7.9 \%(n=6)$, and at 20 $\mathrm{ms}$, depression was complete in some animals with the mean value being $11.97 \pm 6.59 \%(n=5)$. For the Gs heteronymous reflex, the test response was $42.08 \pm$ $4.95 \%$ at $200 \mathrm{~ms}(n=5)$ but $110.43 \pm 46.59 \%$ at $20 \mathrm{~ms}$ $(n=5)$. This suggests that, in contrast to the $\mathrm{H}$ reflex, the heteronymous Gs reflex shows depression at lower stimulation frequencies but is more likely to be facilitated at higher frequencies. This is consistent with the work of Eccles and Rall (1951), who showed that paired homonymous stimulation resulted in reflex facilitation at very short intervals and depression at longer stimulus intervals. Early facilitation of the heteronymous reflex likely reflects a summation of stimuli at afferent collateral branch points, thereby reducing transmission failures (Wall and McMahon, 1994).

\section{Cortical conditioning stimuli exhibit distinct modulation of homonymous and heteronymous MSR}

Next, we determined the effects of cortical conditioning stimuli on the amplitude of the MSR. To do this, we used sub-threshold cortical stimulation to ensure that both inhibition and facilitation of the MSR were possible (Fig. $3 A$ ). Supra-threshold stimulation would result in cortical MEPs, which are likely to mask the effects of interposed inhibitory pathways to motoneurons.

Cortical stimuli preceding plantar nerve stimulation by $20 \mathrm{~ms}$ resulted in either facilitation or inhibition of the IF muscle $\mathrm{H}$ reflex (Fig. 3B-E'). Facilitation was seen both when IF $\mathrm{H}$ wave was supra- and sub-threshold. Interestingly, we observed no obvious laterality for $\mathrm{Cx}$ facilitation of IF $\mathrm{H}$ reflex (contralateral facilitation $=4 / 6$ rats, ipsilateral facilitation $=4 / 8$ rats) but inhibition was less likely for the contralateral ( $2 / 6$ rats) compared to the ipsilateral CST (4/8 rats; Fig. 3B,C). It is widely appreciated that the CST is mainly a crossed pathway and that MEPs are rarely evoked ipsilaterally (Neafsey et al., 1986). Indeed, in our preparation, supra-threshold cortical stimulation only produced MEPs in the contralateral limbs. Surprisingly however, we found that cortical stimulation always resulted in bilateral modulation of both homonymous and heteronymous MSRs. Moreover, we found that $100 \%$ of heteronymous Gs MSRs were strongly facilitated by ipsilateral and contralateral cortical stimulation. This included both threshold reduction at sub-threshold stimulus intensities, and increased response amplitude at supra-threshold intensities. These results suggest a lack of laterality for cortical modulation of lumbar proprioceptive reflexes.

\section{MRF stimulation differentially modulates ipsi- and contralateral intrinsic foot muscle $\mathrm{H}$ reflexes}

We were also able to test bulbospinal modulation of lumbar proprioceptive reflexes by administering conditioning stimuli within the MRF (Fig. 4A). MRF stimulation, also preceding MSRs by $20 \mathrm{~ms}$, resulted in both inhibition and facilitation of the IF $\mathrm{H}$ reflex. For ipsilateral conditioning stimuli, either facilitation or inhibition of the $\mathrm{H}$ reflex was seen at a frequency of $50 \%$ (Fig. 4B,C). For contralateral conditioning stimuli, plantar $\mathrm{H}$ reflexes were more likely to be facilitated (5/7) than inhibited (2/7). Similar to cortical conditioning, heteronymous Gs MSRs were facilitated bilaterally in $100 \%$ of animals.

\section{Discussion}

This study describes a relatively non-invasive protocol for recording bilateral reflexes across two different joints via simultaneous activation of homonymous IF muscle afferents and their heteronymous collaterals to Gs motoneurons. Modulation of these pathways can be assessed by conditioning stimuli to supraspinal structures such as the motor cortex and medullary reticular formation. Our results show that IF homonymous and Gs heteronymous reflexes are differentially modulated by supraspinal conditioning stimuli. IF $\mathrm{H}$ reflexes were modulated bidirectionally while Gs MSRs were facilitated only. Remarkably, we show that cortical modulation of these reflexes lacks the heavily weighted laterality predicted from previous anatomic and MEP physiologic studies.

Proficient control of movement involves the coordination of many synergistic and antagonistic muscles bilaterally. Much of our knowledge regarding sensory contributions to movement control in healthy and injured states come from the assessment of afferent input to single muscles. However, it is well appreciated that low threshold la PA make monosynaptic connections with their homonymous motoneurons as well as many other heteronymous, synergist motoneurons (Baldissera et al., 1981; Hultborn, 2006). This has been demonstrated in experiments using both electrical stimulation of peripheral nerves and stretch activation of muscle spindles (Eccles et al., 1957; Edgley et al., 1986; Wilmink and Nichols, 2003). Heteronymous afferent connections are important for establishing and coordinating muscle synergies in motor control, but are rarely assessed in normal or dysfunctional states. Recent data have reinforced the importance for greater understanding of afferent plasticity in health and disease, beyond that of direct homonymous connections. For example, Mendelsohn et al. (2015) used a clever genetic strategy to show that that the formation of heteronymous afferent terminals on synergist MNs is activity dependent. Similarly, establishment of normal PA input to Renshaw cells (presumably from collateral branches) is dependent on activity from descending systems during postnatal development (Smith et al., 2017). Despite the clear need for development of such knowledge, the lack of a well-defined technique for routinely assessing heteronymous afferent systems has proved a significant barrier to progression. 


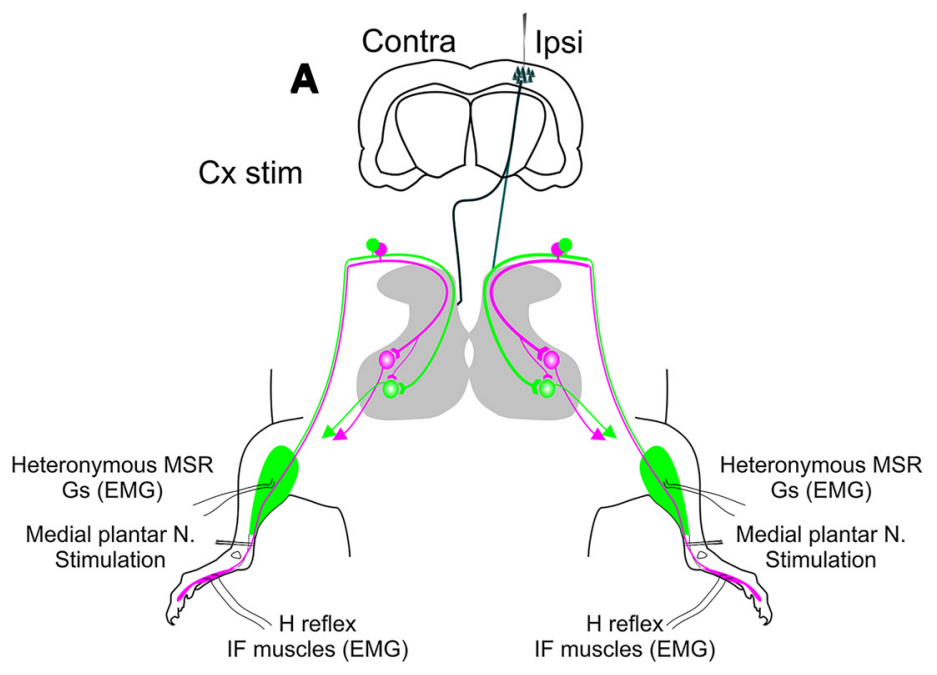

B

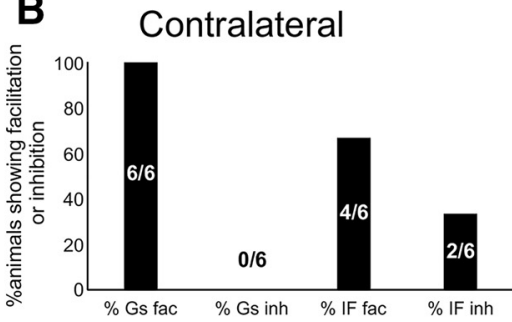

C

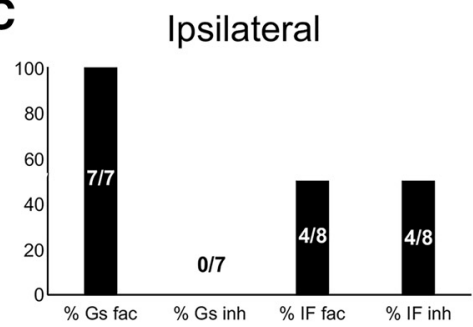

B'

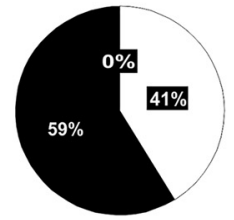

\%Gs Fac IF Inh $\mathbf{C}^{\prime}$

$\%$ both Fac

\% IF Fac Gs Inh

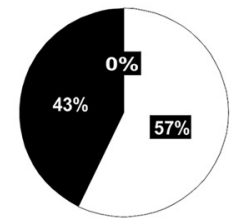

D' Example both facilitated

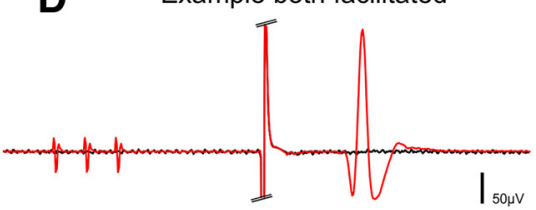

E'

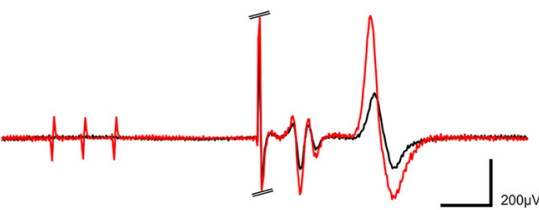

Figure 3. Cortical modulation of homo/heteronymous reflexes bilaterally. A, Schematic of the experimental set up. Cortex was stimulated ipsilaterally and conditioning pulses preceded either ipsi- or contralateral medial plantar nerve stimulation by 20 ms. $\boldsymbol{B}, \boldsymbol{C}$, Percentage of animals in which Cx conditioning stimuli induced facilitation of both Gs and IF reflexes or facilitated Gs but inhibited IF. $\boldsymbol{D}, \boldsymbol{E}$, Example of animal in which Gs is facilitated and IF inhibited. $\boldsymbol{D}^{\prime}, \boldsymbol{E}^{\prime}$, Example of animal in which both Gs and IF reflexes are facilitated. Double lines at the end of a stimulus artifact represent truncation for visual purposes.

This study overcomes that barrier by describing a method to record heteronymous, synergistic MSRs in the Gs while recording $\mathrm{H}$ reflexes in the IF muscles. It therefore confers a major advancement in the capability to investigate mechanisms contributing to motor dysfunction and recovery in the spinal cord. First, the protocol allows recording of MSRs bilaterally, which is significant considering the bilateral nature of mammalian motor control, especially locomotion. Second, simultaneously recording heteronymous MSRs across different joints permits investigation of muscle synergy interactions in the healthy spinal cord, disease or injury states, and after 


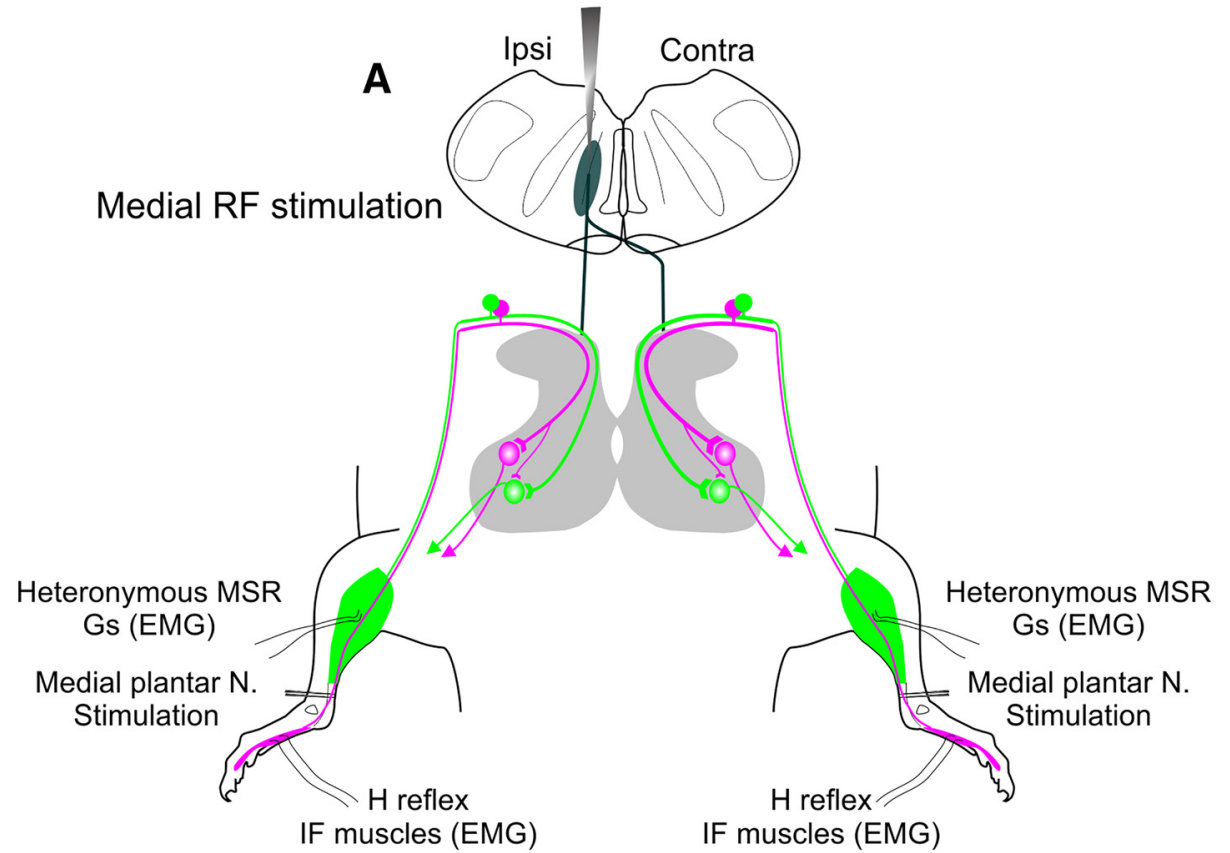

B
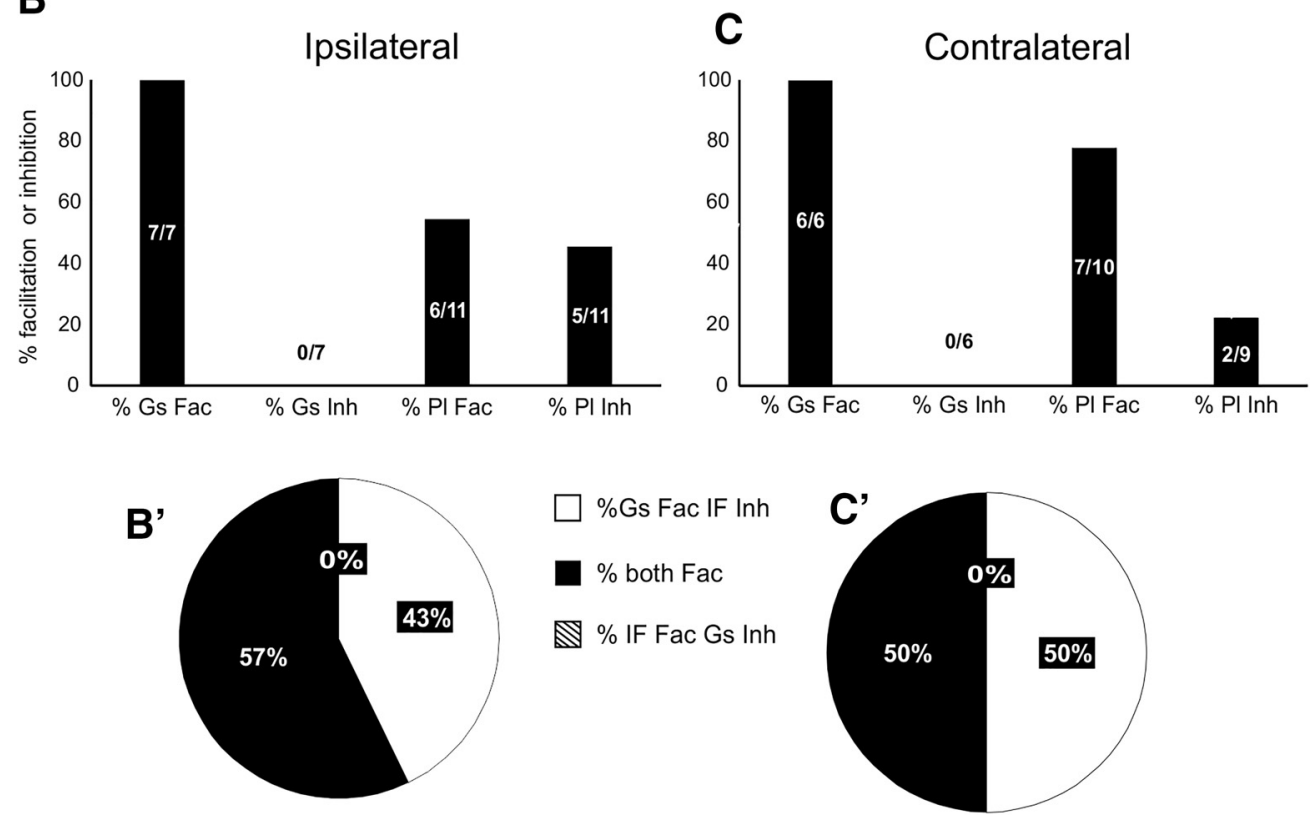

Figure 4. Modulation of bilateral homonymous and heteronymous reflexes by stimulation of the MRF. $\boldsymbol{A}$, Schematic of the experimental set up. MRF was stimulated ipsilaterally and conditioning pulses preceded either ipsi- or contralateral medial plantar nerve stimulation by $20 \mathrm{~ms}$. $\boldsymbol{B}, \boldsymbol{C}$, Percentage of animals in which MRF conditioning stimuli induced facilitation or inhibition of Gs and IF MSRs.

therapy. Finally, efficient interaction with the environment necessitates functionality in both gross and fine motor tasks. Specific training is needed to promote recovery of such tasks and often training of one task can be detrimental to the recovery (negative transfer) of another (De Leon et al., 1999; Marsh et al., 2011). Our method allows afferent control of motor output to be assessed at proximal muscles involved in gross tasks such as locomotion as well as smaller distal muscles, which are associated with skilled tasks such as object manipulation and climb- ing. We propose that future studies assessing plasticity of PA following lesions to descending systems and subsequent therapies, should use bilateral, multi-joint assessments of PA function to provide further insight into potential mechanisms of motor dysfunction and recovery.

Interestingly, we show that cortical and MRF conditioning stimuli always facilitated bilateral Gs MSRs, but homonymous IF foot $\mathrm{H}$ reflexes could be either inhibited or facilitated. This makes sense functionally, as greater supraspinal control over the smaller distal musculature of 
the hind paws, which control digit movements, may be more advantageous for tasks such as climbing and object manipulation compared to the mainly locomotor function of the gastrocnemius muscles.

Facilitation of reflexes could occur via several mechanisms. First, PA terminals are subject to presynaptic inhibition (PSI) via GABApre interneurons, which receive both excitatory and inhibitory modulation from supraspinal systems (Rudomin and Schmidt, 1999). It has previously been demonstrated that cortical conditioning stimuli facilitate $\mathrm{H}$ reflexes in the hindlimb of humans via reduced PSI; therefore, disinhibition of tonic GABApre-mediated PSI may be responsible for facilitation of the MSR (Iles, 1996). Similarly, opposing effects of supraspinal stimulation on both reflexes might be due to increased PSI of IF PA in conjunction with increased excitatory drive to Gs. As rodents lack direct cortico-motoneuronal connections, the CST innervates intermediate excitatory pre-motor INs in the spinal cord which are responsible for cortical evoked MEP responses. (Ueno et al., 2018). It is possible that facilitation of Gs MSR may occur via summation of PA and CST excitation of the intermediate INs involved in eliciting cortical MEPs.

We hypothesize that the reason Gs heteronymous MSRs were always facilitated by conditioning stimuli to $\mathrm{Cx}$ and MRF is related to a reduction in branch point failures in IF heteronymous collateral fibers. These failures in action potential transmission are common, especially in long intersegmental branch projections such as those from IF afferents (mainly L5) to Gs MNs (L4-L5; Nicolopoulos-Stournaras and Iles, 1983; Crockett et al., 1987; Wall and McMahon, 1994). Li et al. (2017) suggested that primary afferent depolarization (PAD), mediated by presynaptic GABApre terminals is able to bring collateral afferents closer to threshold, therefore increasing the probability of AP propagation through presynaptic facilitation (PSF). In agreement, we propose that GABA-mediated PAD acts to produce the classical inhibitory shunt of action potentials in homonymous IF PA but brings branching Gs heteronymous collaterals closer to threshold for AP generation, meaning that IF $\mathrm{H}$ reflexes are inhibited while Gs MSRs are facilitated. Although we suggest this as a likely mechanism, further studies are needed to confirm the hypothesis.

Our most surprising finding was that cortical conditioning of bilateral homonymous and heteronymous PA reflexes showed a lack of laterality in modulation. Although the mainly contralateral (5\% ipsilateral, 95\% contralateral) projecting CST is the most direct pathway, the cortex has diffuse and divergent projections to many subcortical and spinal nuclei which subsequently impinge on spinal motor and premotor neurons (Lemon, 2008). Our results support the idea that cortical modulation of spinal circuits is mediated by a "cortical motor system", which includes a relatively small contribution from direct contralateral projecting CST axons compared to the many divergent subcortical projections. Indeed, it has been demonstrated across multiple species that corticospinal tract lesions have minimal effects on locomotor abilities (Lawrence and Kuypers, 1968; Muir and Whishaw, 1999). Although we cannot conclude which pathways mediate the ipsilateral cortical modulation of both heteronymous and homonymous MSRs, we speculate that cortico-bulbar pathways are utilized as bulbo-spinal projections lack strong laterality in rats (Armstrong, 1988). Additionally, the relatively fast conduction velocity of reticulospinal axons could account for synaptic delay in the reticular formation (Alstermark et al., 2004). The mechanism of facilitation of both MSRs would be different for bulbo-spinal projections because they synapse directly on MNs, and could therefore exert their effects by depolarizing the membrane potential, enabling a greater proportion of motor units to be recruited for a given stimulus, or the opposite. However, the bulbo-spinal tract could also use similar mechanisms to the CST such as PSI, PSF and other intermediate spinal premotor interneuron populations (Engberg et al., 1968; Rudomin, 1990; Alstermark et al., 2004).

Our results describe a novel, relatively non-invasive technique for assessing the physiology of homonymous and heteronymous afferent pathways in rats. This protocol will prove particularly useful to researchers aiming to further delineate mechanisms of motor dysfunction and recovery in disease and injury states, beyond what is currently possible with single muscle, homonymous $\mathrm{H}$ reflex assessments.

\section{References}

Alstermark B, Ogawa J, Isa T (2004) Lack of monosynaptic corticomotoneuronal EPSPs in rats: disynaptic EPSPs mediated via reticulospinal neurons and polysynaptic EPSPs via segmental interneurons. J Neurophysiol 91:1832-1839. CrossRef

Armstrong DM (1988) The supraspinal control of mammalian locomotion. J Physiol 405:1-37. Medline

Baldissera F, Hultborn H, Illert M (1981) Integration in spinal neuronal systems. Comprehensive physiology. Hoboken, NJ: John Wiley \& Sons, Inc.

Burkholder TJ, Nicols TR (2000) The mechanical action of proprioceptive length feedback in a model of the cat hindlimb. Motor Control 4:201-220. Medline

Crockett DP, Harris SL, Egger MD (1987) Plantar motoneuron columns in the rat. J Comp Neur 265:109-118. CrossRef Medline

Crone C, Hultborn H, Mazières L, Morin C, Nielsen J, PierrotDeseilligny E (1990) Sensitivity of monosynaptic test reflexes to facilitation and inhibition as a function of the test reflex size: a study in man and the cat. Exp Brain Res 81:35-45. Medline

De Leon R, Tamaki H, Hodgson J, Roy R, Edgerton V (1999) Hindlimb locomotor and postural training modulates glycinergic inhibition in the spinal cord of the adult spinal cat. J Neurophysiol 82:359-369. CrossRef

Eccles JC, Rall W (1951) Effects induced in a monosynaptic reflex path by its activation. J Neurophysiol 14:353-376. CrossRef Medline

Eccles J, Eccles RM, Lundberg A (1957) The convergence of monosynaptic excitatory afferents on to many different species of alpha motoneurones. J Physiol 137:22-50. CrossRef

Edgley S, Jankowska E, McCrea D (1986) The heteronymous monosynaptic actions of triceps surae group la afferents on hip and knee extensor motoneurones in the cat. Exp Brain Res 61:443446.

Engberg I, Lundberg A, Ryall R (1968) Reticulospinal inhibition of interneurones. J Physiol 194:225-236. CrossRef

Ho SM, Waite PM (2002) Effects of different anesthetics on the paired-pulse depression of the $\mathrm{h}$ reflex in adult rat. Exp Neurol 177:494-502. CrossRef 
Hultborn H, Illert M, Nielsen J, Paul A, Ballegaard M, Wiese H (1996) On the mechanism of the post-activation depression of the H-reflex in human subjects. Exp Brain Res 108:450-462.

Hultborn H (2006) Spinal reflexes, mechanisms and concepts: from Eccles to Lundberg and beyond. Prog Neurobiol 78:215-232. CrossRef Medline

lles J (1996) Evidence for cutaneous and corticospinal modulation of presynaptic inhibition of la afferents from the human lower limb. J Physiol 491:197-207. CrossRef

Kathe C, Hutson TH, McMahon SB, Moon LDF (2016) Intramuscular neurotrophin-3 normalizes low threshold spinal reflexes, reduces spasms and improves mobility after bilateral corticospinal tract injury in rats. Elife 5:e18146.

Knikou M (2008) The H-reflex as a probe: pathways and pitfalls. J Neurosci Methods 171:1-12. CrossRef Medline

Lawrence DG, Kuypers HG (1968) The functional organization of the motor system in the monkey. II. The effects of lesions of the descending brain-stem pathways. Brain 91:15-36. CrossRef

Lemon RN (2008) Descending pathways in motor control. Annu Rev Neurosci 31:195-218. CrossRef Medline

Li Y, Lucas-Osama AM, Black S, Fenrich KK, Stephens MJ, Sanelli L, Lin S, Fouad K, Gorassini MA, Bennett DJ (2017) Presynaptic facilitation of the monosynaptic reflex in humans and rats. Society for Neuroscience 47th Annual Meeting, Washington, DC.

Lundberg A, Norrsell U, Voorhoeve P (1962) Pyramidal effects on lumbo-sacral interneurones activated by somatic afferents. Acta Physiol Scand 56:220-229. CrossRef Medline

Marsh BC, Astill SL, Utley A, Ichiyama RM (2011) Movement rehabilitation after spinal cord injuries: emerging concepts and future directions. Brain Res Bull 84:327-336. CrossRef

Meinck HM (1976) Occurrence of the $\mathrm{H}$ reflex and the $\mathrm{F}$ wave in the rat. Electroencephalogr Clin Neurophysiol 41:530-533. Medline

Mende M, Fletcher EV, Belluardo JL, Pierce JP, Bommareddy PK, Weinrich JA, Kabir ZD, Schierberl KC, Pagiazitis JG, Mendelsohn AI, Francesconi A, Edwards RH, Milner TA, Rajadhyaksha AM, van Roessel PJ, Mentis GZ, Kaltschmidt JA (2016) Sensory-derived glutamate regulates presynaptic inhibitory terminals in mouse spinal cord. Neuron 90:1189-1202.

Mendelsohn Al, Simon CM, Abbott L, Mentis GZ, Jessell TM (2015) Activity regulates the incidence of heteronymous sensory-motor connections. Neuron 87:111-123. CrossRef

Muir GD, Whishaw IQ (1999) Complete locomotor recovery following corticospinal tract lesions: measurement of ground reaction forces during overground locomotion in rats. Behav Brain Res 103:45-53. CrossRef

Neafsey E, Bold E, Haas G, Hurley-Gius K, Quirk G, Sievert C, Terreberry $R$ (1986) The organization of the rat motor cortex: a microstimulation mapping study. Brain Res 396:77-96. CrossRef

Nelson SG, Mendell LM (1978) Projection of single knee flexor la fibers to homonymous and heteronymous motoneurons. J Neurophysiol 41:778-787. CrossRef Medline

Nicolopoulos-Stournaras S, Iles JF (1983) Motor neuron columns in the lumbar spinal cord of the rat. J Comp Neur 217:75-85. CrossRef

Nielsen J, Crone C, Hultborn H (2007) The spinal pathophysiology of spasticity-from a basic science point of view. Acta Physiologica 189:171-180. CrossRef Medline

Pearson KG (1995) Proprioceptive regulation of locomotion. Curr Opin Neurobiol 5:786-791. Medline

Rudomin P (1990) Presynaptic inhibition of muscle spindle and tendon organ afferents in the mammalian spinal cord. Trends Neurosci 13:499-505. Medline

Rudomin P, Schmidt RF (1999) Presynaptic inhibition in the vertebrate spinal cord revisited. Exp Brain Res 129:1-37. CrossRef

Smith CC, Paton JF, Chakrabarty S, Ichiyama RM (2017) Descending systems direct development of key spinal motor circuits. J Neurosci 37:6372-6387.

Takeoka A, Vollenweider I, Courtine G, Arber S (2014) Muscle spindle feedback directs locomotor recovery and circuit reorganization after spinal cord injury. Cell 159:1626-1639.

Tan AM, Chakrabarty S, Kimura H, Martin JH (2012) Selective corticospinal tract injury in the rat induces primary afferent fiber sprouting in the spinal cord and hyperreflexia. J Neurosci 32:1289612908. CrossRef

Ueno M, Nakamura Y, Li J, Gu Z, Niehaus J, Maezawa M, Crone SA, Goulding M, Baccei ML, Yoshida Y (2018) Corticospinal circuits from the sensory and motor cortices differentially regulate skilled movements through distinct spinal interneurons. Cell Rep 23: 1286-1300.e7. CrossRef

Wall PD, McMahon S (1994) Long range afferents in rat spinal cord. III. Failure of impulse transmission in axons and relief of the failure after rhizotomy of dorsal roots. Phil Trans R Soc Lond B 343:211223.

Wilmink RJ, Nichols TR (2003) Distribution of heterogenic reflexes among the quadriceps and triceps surae muscles of the cat hind limb. J Neurophysiol 90:2310-2324. CrossRef Medline 